\title{
ARTICLE
}

\section{Inhomogeneous spatial distribution of the magnetic transition in an iron-rhodium thin film}

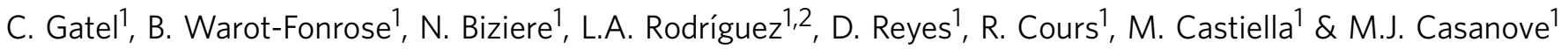

Monitoring a magnetic state using thermal or electrical activation is mandatory for the development of new magnetic devices, for instance in heat or electrically assisted magnetic recording or room-temperature memory resistor. Compounds such as FeRh, which undergoes a magnetic transition from an antiferromagnetic state to a ferromagnetic state around $100{ }^{\circ} \mathrm{C}$, are thus highly desirable. However, the mechanisms involved in the transition are still under debate. Here we use in situ heating and cooling electron holography to quantitatively map at the nanometre scale the magnetization of a cross-sectional FeRh thin film through the antiferromagnetic-ferromagnetic transition. Our results provide a direct observation of an inhomogeneous spatial distribution of the transition temperature along the growth direction. Most interestingly, a regular spacing of the ferromagnetic domains nucleated upon monitoring of the transition is also observed. Beyond these findings on the fundamental transition mechanisms, our work also brings insights for in operando analysis of magnetic devices. 
T he FeRh alloy presents a remarkable and unusual magnetic transition from a low temperature antiferromagnetic state (AFM) to a high temperature ferromagnetic state (FM) close to $370 \mathrm{~K}$ accompanied by a $1 \%$ volume expansion ${ }^{1-5}$. The transition is obtained for a narrow composition range $0.48<x<0.56$ in the B2-ordered $\alpha^{\prime}$ crystal phase of $\mathrm{Fe}_{1-x} \mathrm{Rh}_{x}$. Its main characteristics have been studied both theoretically ${ }^{6-8}$ and experimentally using dedicated techniques such as Mössbauer ${ }^{9,10}$, neutrons ${ }^{3,11,12}$ and XMCD ${ }^{13-18}$. These remarkable features make FeRh particularly well suited for advanced magnetic devices ${ }^{19}$ for instance in heat-assisted or even electrically assisted magnetic recording ${ }^{20-22}$ and magnetic random access memories based on AFM spintronics ${ }^{23}$, or for magnetocaloric materials ${ }^{24}$. Further developments of reliable devices including thin FeRh films or others materials presenting a magnetic transition (as $\mathrm{MnAs}^{25,26}$ ) require a full control of the magnetic state within the film, a perfect knowledge of the mechanisms involved in the transition and a deep understanding of the influence of interfaces and low-dimensionality.

Different publications reported the persistence of an FM component at room temperature or even below in ultrathin FeRh films ${ }^{27,28}$ or at the interface between the film and the capping layers ${ }^{12,14,29,30}$. These observations were attributed to chemical diffusion, surface segregation or misfit strain, and often to a combination of these different effects. The role of surface or interface in the nucleation of the FM domains at the onset of the transition was also analysed experimentally $17,18,29-31$. Few studies have mapped the transition on thin films ${ }^{17,18,30}$. They report co-existing FM and AFM domains on the top surface and the subsequent expansion of the FM domains. In addition, the magnetic profile along the growth direction of FeRh films, for both undoped and doped films, was analysed by polarized neutron reflectometry with a sub-nm resolution ${ }^{12,29,32,33}$. Spectra were suitably fitted by taking into account both a top and a bottom interface regions (around $8 \mathrm{~nm}$ thick) surrounding the core of the film.

Here we report quantitative imaging on the local magnetization by electron holography (EH). In contrast with the previous experimental methods, this technique is highly sensitive to the magnetic signal ${ }^{34-37}$ and information can be extracted across the entire film thickness with unrivalled spatial resolution, notably along the interfaces, compared to polarized neutron reflectometry. In situ EH experiments were performed on a cross-sectional $50 \mathrm{~nm}$ thick FeRh film to fully investigate the magnetic transition mechanism at a nanometre scale, with the objective to unravel the different effects attributed to size and interfaces. We first demonstrate that the in situ experiment performed on the TEM specimen, that is, on a very small area, suitably reproduces the macroscopic magnetization loop measured at the AFM-FM transition using vibrating sample magnetometry (VSM). Comparing these local and macroscopic results enables us to provide essential information on the TEM specimen, and on the effect of focused ion beam thinning (FIB) preparation. In a second part, we exploit the magnetic phase images recorded within the film upon heating and cooling cycles to map the evolution of the transition temperature from surface to interface with the substrate and quantify the magnetization changes. Our results provide insight into the transition mechanisms.

\section{Results}

Structural properties of the studied area. A $50 \mathrm{~nm}$ FeRh film was epitaxially grown at $550^{\circ} \mathrm{C}$ on a $\mathrm{MgO}(001)$ substrate by $\mathrm{DC}$ sputtering and in situ annealed for $6 \mathrm{~h}$ at $800^{\circ} \mathrm{C}$. The AFM/FM transition in this film was first studied by VSM. For EH

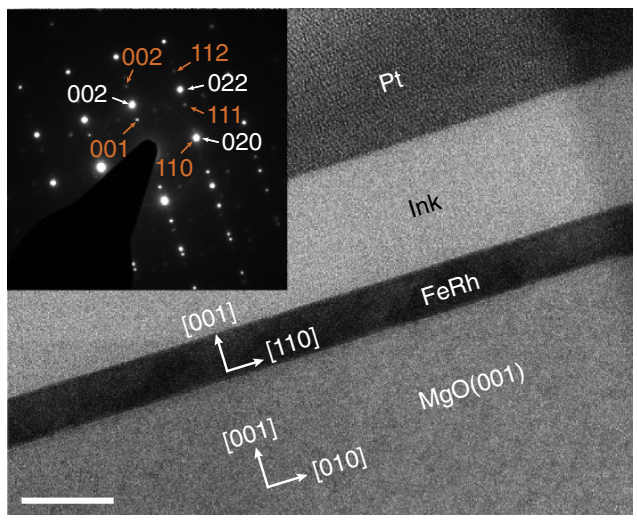

Figure 1 | Epitaxial FeRh thin film on MgO(001) substrate. Conventional TEM image (main panel) and diffraction pattern (inset) of the area studied by electron holography. The scale bar is $100 \mathrm{~nm}$. On the diffraction pattern, indexes in orange correspond to the FeRh layer and white ones to the $\mathrm{MgO}$ substrate.

experiments, a cross-sectional lamella was prepared by FIB to ensure a uniform thickness crossed by the electron beam. The FeRh layer was protected by ink and platinum layers to avoid damages and charge accumulation during the thinning process. The final thickness of the lamella was measured at $90 \pm 5 \mathrm{~nm}$ after a low energy step to minimize irradiation damages and surface amorphization (see Supplementary Fig. 1).

Figure 1 presents a TEM image of the area used for the EH study. The corresponding diffraction pattern demonstrates the epitaxial growth of the monocrystalline FeRh layer on the substrate with a $45^{\circ}$ rotation between the FeRh bcc lattice and the $\mathrm{MgO}$ fcc lattice. The growth axis is [001] and the layer is observed along the FeRh [-110] axis ([100] for $\mathrm{MgO}$ ). The appearance of the 001 reflections reveals the $\mathrm{B} 2$ ordered phase.

Series of holograms and principle of the data analysis. The basic principles of $\mathrm{EH}$ and the description of the full procedure for the magnetic phase shift extraction as well as for the magnetization quantification are detailed in Supplementary Notes 4 and 5. In the following, [110] and [001] directions in $\mathrm{FeRh}$ are chosen as $x$ axis and $y$ axis respectively. The direction parallel to the electron beam stands for the $z$ axis. Using the Aharanov-Bohm equations ${ }^{38,39}$, the $x$ component of the induction, noted $B_{x}$, was extracted from a defined area of the magnetic phase image by analysing the mean slope of the magnetic phase shift $\phi_{\mathrm{M}}$ along the $y$ direction:

$$
\int B_{x}(x, y, z) \mathrm{d} z=\frac{\hbar}{e} \frac{\partial \phi_{\mathrm{M}}(x, y)}{\partial y}
$$

with $e$ the electron charge and $\hbar$ the reduced Planck constant. Measuring the lamella width $w$ crossed by the electron beam and assuming that the magnetization is equivalent to the induction inside the FeRh layer, that is, neglecting the stray fields, it is then possible to obtain the $x$ component of the magnetization, noted $\mu_{O} M_{x}$ :

$$
\mu_{0} M_{x}=\frac{\int B_{x}(x, y, z) \mathrm{d} z}{w}
$$

Repeating this analysis as a function of the applied temperature $T$, we then can plot $M_{x}=f(T)$ on a very localized area. The resulting graph is therefore strictly equivalent to the classical $M(T)$ curve obtained by magnetometry.

Prior to the EH experiment, the FeRh layer was slightly tilted away from the $[-110]$ zone axis to avoid dynamical diffraction effects which create artefacts on the phase measurement. The 

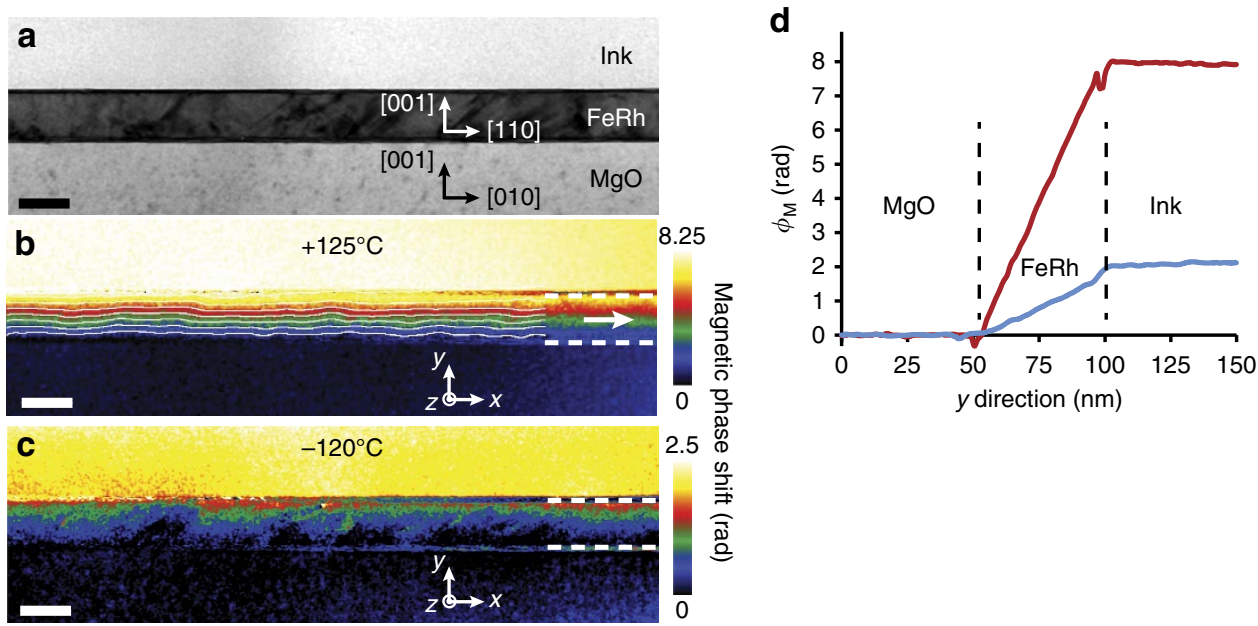
phase image obtained at $+125^{\circ} \mathrm{C}$. The FeRh layer is between horizontal dashed lines at the right of the image. The white arrow and isophase lines parallel to the magnetic induction lines into the FeRh layer have been added for clarity. (c) Magnetic phase image obtained at $-120^{\circ} \mathrm{C}$. (d) Magnetic phase shift profiles along the $y$ direction averaged over the $x$ direction for the whole field of view. The red and blue curves correspond to the profiles extracted at $+125^{\circ} \mathrm{C}$ and $-120^{\circ} \mathrm{C}$ respectively. Scale bars represent $50 \mathrm{~nm}$.

sample temperature was then stabilized at $+125^{\circ} \mathrm{C}$ to get the FeRh layer into the FM state before applying an in situ magnetic field to saturate the specimen parallel to the [110] direction of FeRh (see Supplementary Note 3). A first set of 20 holograms was acquired during a temperature decrease down to $-120^{\circ} \mathrm{C}$ (cooling process) before recording a second set of 35 holograms during the heating process up to $+125^{\circ} \mathrm{C}$. A third set of 22 holograms was recorded by decreasing the temperature down to $-120^{\circ} \mathrm{C}$ but after in situ magnetic saturation at $+125^{\circ} \mathrm{C}$ in the direction opposite to the previous one. All the holograms were acquired at the remnant state on the same area after temperature stabilization to perform a static study of the transition. The smallest temperature step between two successive holograms was $5{ }^{\circ} \mathrm{C}$. We checked between the beginning and the end of the full $\mathrm{EH}$ experiment that the FeRh layer has not been modified by the irradiation of the electron beam by comparing the amplitude images, but also the magnetic phase images obtained in the same ferromagnetic state at $+125^{\circ} \mathrm{C}$.

Magnetic properties for the two extreme temperatures. Figure 2 presents the amplitude and magnetic phase images for +125 and $-120^{\circ} \mathrm{C}$. The field of view of the studied area is $630 \mathrm{~nm} \times 190 \mathrm{~nm}$. The contrast variations visible on the amplitude image (Fig. 2a) are commonly observed by TEM on FeRh layers and are attributed to the presence of defects as dislocations.

The ferromagnetic state clearly shows up on the magnetic phase image obtained at $+125^{\circ} \mathrm{C}$ with the appearance of a phase shift inside the FeRh layer (Fig. 2b). The magnetic induction lines displayed by the isophase contours parallel to the $x$ direction demonstrate an induction aligned along the layer plane. As detailed in Supplementary Note 5, $3 \mathrm{~nm}$ thick layers of FeRh close to the top and bottom interfaces were affected by artefacts due to data processing and consequently were excluded from the analysis. The phase shift profile across the FeRh layer (that is, parallel to the $y$ direction) averaged over the whole field of view in the $x$ direction is given in Fig. $2 \mathrm{~d}$ : the plateau in the ink and $\mathrm{MgO}$ areas indicate that there is no induction outside the film although the linear variation inside the layer proves that the induction is spread homogeneously within the FeRh film. This profile is the same for each position along the $x$ axis and confirms that the magnetization is homogeneous all over the observed area.

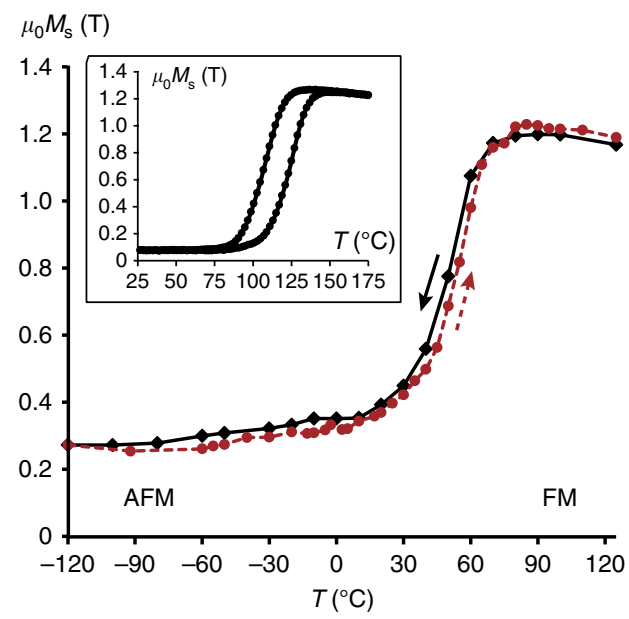

Figure 3 | Local magnetization versus temperature loop. $x$ component of the magnetization as a function of temperature for a complete temperature cycle. The values are obtained at the remnant state from the slope of the magnetic phase shift averaged on the field of view detailed in Fig. 2. The cooling and heating series correspond to the black and red dashed lines respectively. In insert, the $M(T)$ loop measured by vibrating sample magnetometry on the whole FeRh sample from which the thin lamella has been extracted for electron holography experiments. This loop has been acquired with a $2 \mathrm{kOe}$ field applied along the [110] axis of the FeRh layer.

According to equation (1), the integrated induction averaged over all the FeRh layer displayed in Fig. 2a can be obtained from the mean slope of the phase shift profile using a linear fit. The constant slope of $0.163 \pm 0.01 \mathrm{radnm}^{-1}$ in the FeRh layer corresponds therefore to an integrated induction of $107 \pm 6 \mathrm{~T} \mathrm{~nm}$ distributed homogenously in the film thickness. The width $w$ of the TEM lamella being $90 \pm 5 \mathrm{~nm}$, the magnetization $\mu_{0} M_{x}$ was calculated equal to $1.19 \pm 0.13 \mathrm{~T}$ (equation (2)). This value is in very good agreement with previous magnetization values obtained at the FM state ${ }^{10,12,27}$.

The magnetic phase image recorded at $-120^{\circ} \mathrm{C}$ (Fig. 2c) shows a strong decrease of the total phase shift. From its mean slope extracted on the whole FeRh layer, we obtained a residual 


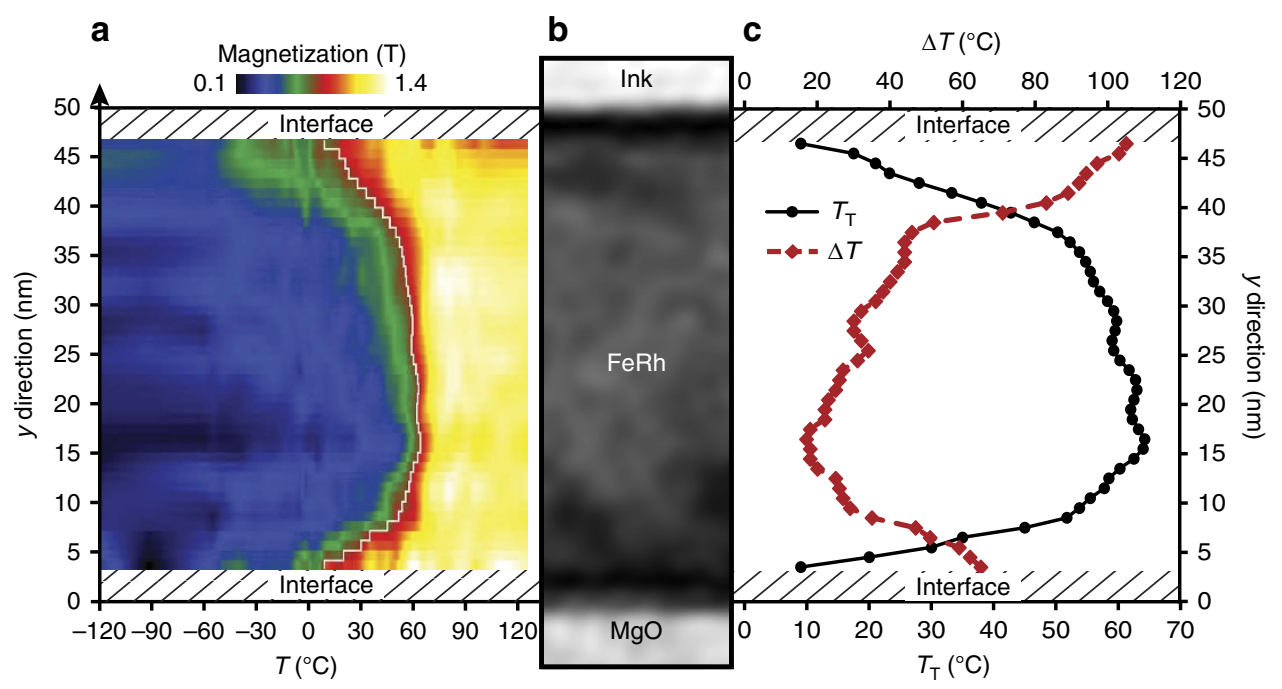

Figure 4 | Evolution of the magnetization in the growth direction. (a) Map of the magnetization as a function of the temperature and the position in the layer depth using the heating temperature series. The colour scale corresponds to the magnitude of the magnetization and the transition temperature is displayed by the white profile corresponding to magnetization of $0.75 \mathrm{~T}$. (b) Amplitude image of the part of the FeRh layer used for the calculation.

(c) Profiles of the transition temperature $T_{T}$ and the transition width $\Delta T$ as a function of the position within the FeRh layer.

magnetization parallel to the film equal to $0.28 \pm 0.13 \mathrm{~T}$. This value appears to be higher than previously reported ${ }^{14,40}$.

Local magnetization versus temperature loop. In order to estimate the influence of the FIB preparation on the magnetic behaviour of the FeRh film, a magnetization versus temperature loop was acquired by $\mathrm{EH}$ and compared to VSM results. The analysis of the phase shift slope was therefore repeated on the same area of the FeRh layer displayed in Fig. 2 both when decreasing (cooling) and increasing (heating) the temperature. Figure 3 shows the complete loop $M_{x}=f(T)$ in the temperature range $-120^{\circ} \mathrm{C}<T<125^{\circ} \mathrm{C}$. Error bars are not reported for better clarity of the plot and are in the range of \pm 0.07 and $\pm 0.1 \mathrm{~T}$ at each point. While increasing the temperature, a plateau is observed around $0.28 \mathrm{~T}$ up to $-40^{\circ} \mathrm{C}$. The magnetization increases slightly up to $20^{\circ} \mathrm{C}$ then with a higher slope up to $45^{\circ} \mathrm{C}$. An even steeper increase occurs up to $65^{\circ} \mathrm{C}$ before slowing down up to a magnetization maximum just over $1.23 \mathrm{~T}$ at $80^{\circ} \mathrm{C}$. Beyond $80^{\circ} \mathrm{C}$, the magnetization follows a Curie-Weiss law usually observed in ferromagnetic materials. The trend is inversed when the temperature is decreased with a slight hysteresis of about $5{ }^{\circ} \mathrm{C}$ between the two curves. The width of the transition is about $60^{\circ} \mathrm{C}$ for heating and cooling, indicating the coexistence of AFM and FM domains over a wide temperature range. By defining the transition temperature $T_{\mathrm{T}}$ as the one corresponding to a magnetization of $0.75 \mathrm{~T}$ (intermediate value between AFM and FM states), $T_{\mathrm{T}}$ is found equal to $55^{\circ} \mathrm{C}\left( \pm 5^{\circ} \mathrm{C}\right)$. The loop opening is measured at about $5^{\circ} \mathrm{C}$ with an uncertainty of $\pm 5^{\circ} \mathrm{C}$ if error bars are taken into account.

The inset in Fig. 3 presents the $M(T)$ loop measured by VSM on the whole FeRh layer from which the TEM lamella has been extracted. A $2 \mathrm{kOe}$ magnetic field was applied during the VSM experiment along the same [110] direction as the one selected for induction measurements ( $x$ direction). Important quantitative differences between the macroscopic and microscopic analyses are evidenced. As explained in the following, this result most probably originates from the difference in both investigated volume and sample geometry in the two techniques. Nevertheless, as in any TEM study, we cannot totally rule out a possible bias due to unexpected local inhomogeneities in the $\mathrm{EH}$ investigated region. First, the transition rate, defined as $\left(M_{\mathrm{HT}}-M_{\mathrm{LT}}\right) / M_{\mathrm{HT}}$ where $M_{\mathrm{HT}}$ is the high-temperature magnetization (FM state) and $M_{\mathrm{LT}}$ is the residual magnetization at low temperature (AFM state), is $94 \%$ when measured macroscopically by VSM and $76 \pm 11 \%$ when using the magnetization obtained by $\mathrm{EH}$ at $+125^{\circ} \mathrm{C}$ and $-120^{\circ} \mathrm{C}$. In addition, the transition temperature is about $108^{\circ} \mathrm{C}$ in the macroscopic sample and about $55^{\circ} \mathrm{C}$ in the thin lamella. Last, the cycle opening reaches $18^{\circ} \mathrm{C}$ in the macroscopic film, while it is about $5^{\circ} \mathrm{C}$ in the $\mathrm{EH}$ specimen.

As the macroscopic magnetization measured in the FM state by VSM is very similar to the one measured locally by $\mathrm{EH}\left(\mathrm{M}_{\mathrm{HT}}=1.27 \mathrm{~T}\right.$ and $1.19 \mathrm{~T}$ respectively $)$, the difference in transition rate comes from the low temperature behaviour (that is, $M_{\mathrm{LT}}$ ): a large FM component remains at low temperature in the TEM specimen used for $\mathrm{EH}$ experiments. Indeed, during the lamella preparation by FIB, the ion beam, even at low energy, creates two amorphous layers on each side of the thin lamella. The FM to AFM transition upon cooling, only present in the $\mathrm{B} 2$ phase $\mathrm{e}^{40}$, is impeded in these regions which remain in the FM state. Assuming a minimum thickness of $5 \mathrm{~nm}$ for each layer, these two damaged FM layers correspond to more than $10 \%$ of the total width of the thin lamella crossed by the electron beam (see Supplementary Fig. 5). The magnetization of the AFM state limited to the B2 phase thickness $(80 \mathrm{~nm})$ is therefore equal to $0.166 \mathrm{~T}$ giving a minimum conversion rate of $86 \pm 13 \%$, that is, in reasonable agreement with the transition rate measured by VSM and others studies. This conversion rate would increase for thicker damaged layers with a magnetization of the AFM state approaching 0 . The difference between the two transition temperatures measured by VSM and EH can also be attributed to the presence of the FIB damaged regions, which decrease the magnetic transition temperature due to their strong magnetic coupling. The small cycle opening is a mark of a quasi-reversibility of the transition process, which would require less energy. It most probably originates from the nanowire-like geometry (1D system) of the TEM lamella (section of $50 \times 90 \mathrm{~nm}^{2}$ over several $\mu \mathrm{m}$ of length) than to the 2D film measured by VSM. The data recorded in EH thus suitably reproduce the magnetic behaviour of the film when finite-size effects and ion beam damaged regions are taken into account. 


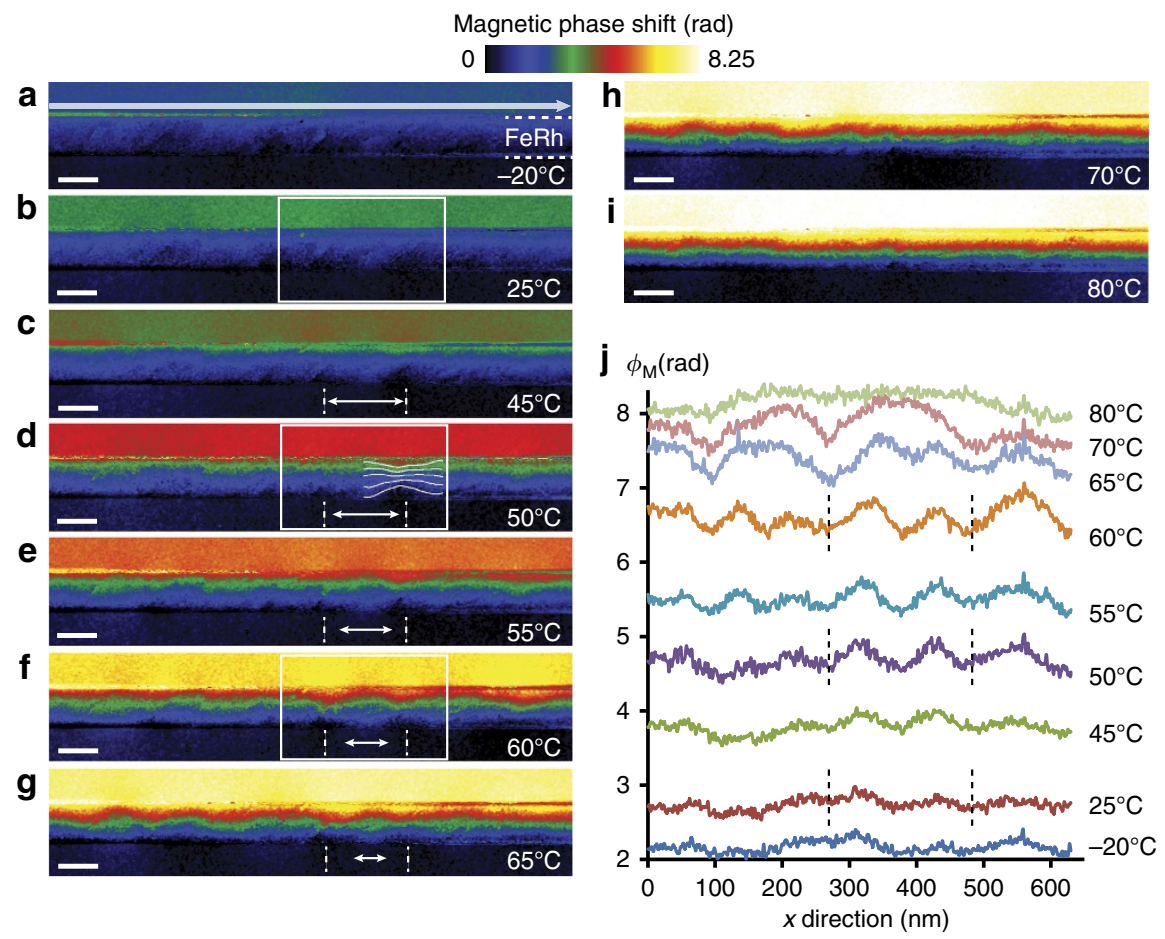

Figure 5 | Main stages of the transition while increasing temperature. (a-i) Magnetic phase images. The colour scale is common to all images and scale bars represent $50 \mathrm{~nm}$. On the phase image recorded at $50^{\circ} \mathrm{C}(\mathbf{d})$ are displayed the isophase lines parallel to the induction lines: the narrowing corresponds to a local increase of the phase variation and the appearance of ferromagnetic domains. The two dotted lines into the MgO part are centred on two ferromagnetic domains and the double arrow between them corresponds to the approximate width of the enclosed antiferromagnetic domain. The areas enclosed by the white rectangles for phase images at $25^{\circ} \mathrm{C}(\mathbf{b}), 50^{\circ} \mathrm{C}(\mathbf{d})$ and $60^{\circ} \mathrm{C}(\mathbf{f})$ are used for micromagnetic simulations presented Fig. 6 . (j) Phase profiles extracted for different temperatures along the upper interface (large arrow on the magnetic phase image obtained at $-20^{\circ} \mathrm{C}$ ).

Evolution of the transition with FeRh layer depth. EH allows for a deeper local analysis of the AFM/FM transition by extracting the magnetic information originating from smaller areas of the FeRh cross-sectional view (see Supplementary Fig. 3b-d). The evolution of the temperature transition along the growth direction has then been studied using a rectangular mask with dimension $x=630 \mathrm{~nm}$ and $y=4 \mathrm{~nm}$ elongated parallel to the interface with the $\mathrm{MgO}$ substrate. The area enclosed by the mask was shifted by $1 \mathrm{~nm}$ along the $y$ direction and, for each position, a complete loop $M_{x}=f(T)$ was extracted. Figure 4a presents the evolution of the AFM/FM transition from the bottom interface to the top surface using the heating temperature series ( 35 holograms from -120 to $+125^{\circ} \mathrm{C}$ ). The first $3 \mathrm{~nm}$ close to both interfaces have been excluded because of artefacts in the data processing. The main result is the evidence of the heterogeneity of the transition: close to the interfaces, especially close to the top surface, the magnetic transition from the AFM state to the FM state starts at much lower temperature and is spread over a wider range of temperature than in the middle of the layer. The profile of $T_{\mathrm{T}}$ along the growth direction has been extracted from Fig. $4 \mathrm{a}$ and is shown in Fig. $4 \mathrm{c}: T_{\mathrm{T}}$ is lower close to the interfaces than in the middle of the layer with a difference up to $50{ }^{\circ} \mathrm{C}$. The core of the layer presents a $30 \mathrm{~nm}$ plateau with a mean value of $T_{\mathrm{T}}$ slightly lower than $60^{\circ} \mathrm{C} . T_{\mathrm{T}}$ decreases towards the interfaces down to $10^{\circ} \mathrm{C}$ over a width of 5 to $10 \mathrm{~nm}$. The transition width $\Delta T$ has been defined as the temperature range corresponding to an integrated induction between 0.5 and $1 \mathrm{~T}$. By superimposing $\Delta T$ and $T_{\mathrm{T}}$ profiles as a function of the position in the film, a clear match occurs: the lower $T_{\mathrm{T}}$, the wider the transition. Similar results are obtained studying the cooling series.

\section{Discussion}

Previous studies have shown for thin films that a FM state was stabilized and the transition temperature measured macroscopically evolved but the origin remained not entirely understood. If the presence of interfaces is considered to be at the origin of these experimental results $27,30,41$, the stress effect ${ }^{10,14,31}$ is also suspected to play a role. The properties of interfaces, in particular with capping layers, have been widely investigated in FeRh films, notably using XMCD-PEEM experiments. The occurrence of a persistent FM state in large surface areas near the surface, depending on the nature of the capping layer, was thus demonstrated ${ }^{12,14,17,29}$. In our case, the interdiffusion of the ink layer is most likely very limited and has no effect, as it was deposited at room temperature and the maximum temperature the system reached is $125^{\circ} \mathrm{C}$. Any structural defects have been seen in the FeRh layer at the top surface compared to the inner part. Similarly, a slight oxidation of the FeRh would spread over less than $2 \mathrm{~nm}$ and has not been observed. So even if we cannot exclude a slight effect of stress, symmetry breaking seems to be the most likely responsible for the magnetic behaviour changes. Regarding the $\mathrm{MgO} / \mathrm{FeRh}$ interface, a diffusion of $\mathrm{Mg}$ might have occurred. In addition, the presence of structural defects such as misfit dislocations at this interface certainly promotes magnetic transition changes. These different mechanisms are sufficient to explain the slight asymmetry of the $T_{\mathrm{T}}$ and $\Delta T$ profiles between both interfaces. We observed also that the interface effects extend up to $15 \mathrm{~nm}$, that is, deeper into layer than previously assumed.

We thus demonstrate that both interfaces have a huge influence on the AFM/FM in the same way by reducing $T_{\mathrm{T}}$ and broadening the temperature range necessary for the transition. This result is of large importance since it explains why various 
experimental measurements observe a decrease of the transition temperature with the decrease of the layer thickness ${ }^{27}$. Our result thus predicts the difficulty to obtain an AFM/FM transition in FeRh nanoparticles of few nanometres diameter. In addition, interface effects have to be taken into account when FeRh layer is integrated in a device and coupled with adjacent (magnetic) layer $20,22,23$.

The magnetic phase images corresponding to the main stages of the transition from low temperatures to high temperatures are presented (Fig. 5). The studied area is identical to the one analysed in Fig. 2 and the colour scale is common to all images. The gradual increase of the total phase shift with increasing temperature reflects the appearance of the FM state as discussed previously. At -20 and $+25^{\circ} \mathrm{C}$, the phase images are relatively similar to the one obtained at $-120^{\circ} \mathrm{C}$ with only slightly larger phase value due to the beginning of the AFM/FM transition at surfaces or interfaces. The magnetic configuration varies more significantly from $45^{\circ} \mathrm{C}$. Interfaces with an almost complete FM state exhibit a higher phase variation (that is, magnetization) than in the core of the film. In addition, inhomogeneities of the phase shift appear in direction parallel to the interfaces ( $x$ direction): some areas with narrow induction lines (that is, corresponding to larger phase variations) become visible within the layer of FeRh (see isophase lines on the phase image recorded at $50^{\circ} \mathrm{C}$ ). They correspond to the nucleation of FM areas. These local FM regions are in addition coupled with other small variations outside the film near the interfaces corresponding to their leak field. In Fig. 5, two FM domains that remain for temperatures between 45 and $65^{\circ} \mathrm{C}$ are identified by dotted lines (drawn in the $\mathrm{MgO}$ part for better clarity). The distance between the cores of these FM domains enclosed within an AFM matrix is about $100 \mathrm{~nm}$. The double white arrow indicates the approximate width of the AFM area between the two FM domains. This width decreases gradually implying a lateral extension of the FM domains before a sudden disappearance of the remaining AFM area between 65 and $70^{\circ} \mathrm{C}$, the coalescence of the FM areas being favoured due to the magnetic coupling between them that promotes the transition from AFM to FM state.

This AFM/FM transition mechanism though FM domain nucleation within the FeRh layer was confirmed by the study of leak fields spreading out of the FM domains. They are evidenced by extracting the magnetic phase profile along the upper interface (white arrow on the phase image at $-20^{\circ} \mathrm{C}$ ). The profiles obtained at different temperatures are given in Fig. $5 \mathrm{j}$ and show the appearance of oscillations whose maxima and minima are centred on the FM and AFM areas respectively. Oscillations appear clearly from $35^{\circ} \mathrm{C}$ (not shown), corresponding to the end of nucleation process, increase in amplitude (FM domain growth) and begin to disappear from $65^{\circ} \mathrm{C}$ (FM domains coalescence) and completely at $80^{\circ} \mathrm{C}$. The most remarkable result is the constant spatial periodicity (of about $100 \mathrm{~nm}$ ) of the FM domains reflecting the regular repetition of the FM and AFM areas. Note that the value of this period is comparable to the distance between misfit dislocations allowing a complete relaxation of a $\mathrm{FeRh}$ layer on $\mathrm{MgO}$. We may assume that the nucleation of these FM domains occur on these structural defects. Our results confirm that the FM phase nucleates at the interfaces and propagates into the bulk as the temperature rises ${ }^{31}$. However we demonstrate the existence of a second mechanism corresponding to the nucleation of small FM domains into the FeRh layer. This mechanism cannot be observed if using probe-based methods.

Micromagnetic simulations with the OOMMF package (http://math.nist.gov/oommf) were used to confirm the appearance of the FM domains in the AFM matrix. These simulations are performed at $0 \mathrm{~K}$, the temperature being introduced through a magnetic model based on by $\mathrm{EH}$ data at a given temperature. In
Fig. 6 we compare the experimental magnetic phase shift at 25, 50 and $60^{\circ} \mathrm{C}$ with the ones calculated from three magnetic configurations. Experimental data were taken from the area marked by a white rectangle in Fig. 5b,d,f. The presence of the disordered FM layers was taken into account (not shown in the $3 \mathrm{D}$ scheme for clarity), as well as the effect of the interfaces on the transition. The distance between the FM domains in the micromagnetic calculations is $100 \mathrm{~nm}$ and their lateral size was adjusted accordingly to the experimental phase images $(20 \mathrm{~nm}$ at $50^{\circ} \mathrm{C}, 40 \mathrm{~nm}$ at $60^{\circ} \mathrm{C}$ ). All other parameters of the simulation are given in Supplementary Note 6. A very good quantitative agreement between the simulated and experimental phase images is obtained for each temperature. No FM domains are observed at $25^{\circ} \mathrm{C}$. At $50^{\circ} \mathrm{C}$, the $\mathrm{FM}$ domains have nucleated within the layer while thickness and magnetization of the ferromagnetic interfaces have increased. At $60^{\circ} \mathrm{C}$, these interfacial areas have completed the transition to the FM state while the FM domains within the layer extend laterally. The small differences occurring between the simulated and experimental phase images is the result of our hypothesis regarding the thickness of the interfacial zones and also may be due to the shape of the FM domains that surely are not perfectly rectangular as proposed.

Successive schemes of the AFM/FM transition have therefore been determined by $\mathrm{EH}$ and observed in the $M(T)$ loop shown in Fig. 3: the induction starts to increase with the interface transition $\left(-40{ }^{\circ} \mathrm{C}<T<20^{\circ} \mathrm{C}\right)$, it accelerates slightly with nucleation of periodic $\mathrm{FM}$ domains from $25^{\circ} \mathrm{C}$ even if the transition at interfaces is not achieved, then an abrupt change from $35^{\circ} \mathrm{C}$ corresponding to the $\mathrm{FM}$ domain growth within the $\mathrm{AF}$ matrix and a slower increase from $65^{\circ} \mathrm{C}$ during the coalescence phase until the complete disappearance of the AFM state. The observations during cooling process show a reverse transition involving the same mechanisms but with a slight shift towards the low temperatures of about $5^{\circ} \mathrm{C}$.

Thus the cross-sectional observation of an FeRh layer at the nanoscale using in situ $\mathrm{EH}$ upon heating and cooling cycles allows us to obtain a clear and quantitative description of the AFM/FM transition process. The analysis of magnetic phase images evidenced an evolution of the transition temperature from the bottom interface with the substrate to the top surface associated to a regular alternation of FM domains in an AF matrix. Our results bring insights for in operando analysis of magnetic devices. Beyond these findings, the paper describes a very promising approach to investigate the mechanisms of phase transitions in various magnetic systems such as $\mathrm{MnAs}^{42}$ at all pertinent scales.

\section{Methods}

Sample growth and thin lamella preparation. The $50 \mathrm{~nm}$ thick FeRh layer was grown on an $\mathrm{MgO}$ (001) substrate by DC sputtering using a co-deposition process from two pure $\mathrm{Fe}$ and $\mathrm{Rh}$ targets. The film was deposited at $550{ }^{\circ} \mathrm{C}$ and in situ annealed for $6 \mathrm{~h}$ at $800^{\circ} \mathrm{C}$. For EH experiments, a cross-sectional lamella was prepared by FIB to ensure a uniform thickness crossed by the electron beam. The FeRh layer was protected by a $150 \mathrm{~nm}$ thick ink layer and a $250 \mathrm{~nm}$ thick Pt layer to avoid damages and charge accumulation during the thinning process. The lamella was then extracted and thinned down to about $100 \mathrm{~nm}$ to get electron transparency with a final step at low energy to minimize irradiation damages and amorphization of the surfaces.

Magnetometry. The AFM/FM transition was checked by VSM of a quantum Design Physical Properties Measurement System (PPMS) on the whole sample elaborated by sputtering. A continuous 2 kOe magnetic field was applied along the in plane [110] direction of FeRh during all magnetization measurements as a function of temperature.

TEM and heating-cooling sample holder. The EH experiment was performed using a Hitachi HF 3300C microscope operating at $300 \mathrm{kV}$. Dedicated Lorentz modes combined with the B-core corrector allow achieving a $0.5 \mathrm{~nm}$ spatial 


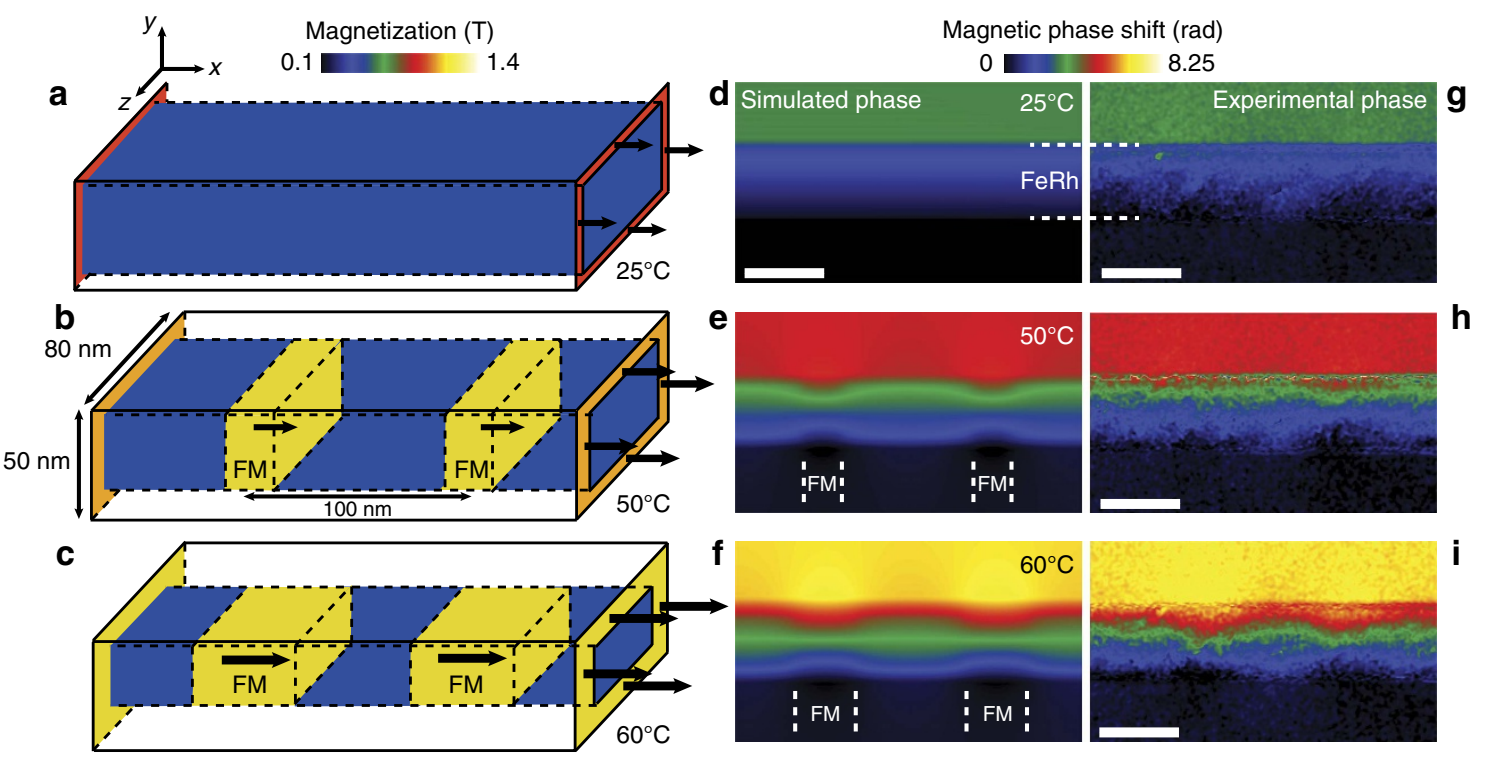

Figure 6 | Micromagnetic simulations and comparison with experimental phase images. (a-c) 3D schemes used for the micromagnetic simulations of the area enclosed by the white rectangle in Fig. $5 b, d, f\left(25,50\right.$ and $60^{\circ} \mathrm{C}$ respectively). The ferromagnetic damaged surfaces are not represented for clarity. (d-i) Comparison between simulated magnetic phase images calculated from micromagnetic simulations and (h-j) experimental magnetic phase images obtained at same temperatures. Scale bars represent $50 \mathrm{~nm}$.

resolution in a field-free environment (less than 10 Oe). All the holograms were recorded in a 2 biprism configuration ${ }^{43}$ with a fringe spacing set to $0.9 \mathrm{~nm}$. The sample holder is a single tilt Gatan HC3500 holder that permits temperature control by PID (Proportional Integral Derivative) from -150 to $+250^{\circ} \mathrm{C}$.

Data treatment. Phase and amplitude images were extracted from the holograms using homemade software based on Fourier analysis. The size of the digital mask used in the FFT was chosen to obtain a spatial resolution of $2.5 \mathrm{~nm}$ on phase and amplitude images. Amplitude images, which remain the same regardless of the applied temperature, were automatically realigned by a cross-correlation method. This realignment was then applied on the phase images. The electrostatic $\phi_{\mathrm{E}}$ and magnetic $\phi_{\mathrm{M}}$ contributions from the total phase shift were separated using the phase images obtained at $125^{\circ} \mathrm{C}$ after having saturated the magnetization of the layer in opposite directions: $\phi_{\mathrm{E}}$ remains the same while $\phi_{\mathrm{M}}$ sign changes. The half sum of these images provides $\phi_{\mathrm{E}}$, which is then subtracted from all the phase images: only $\phi_{\mathrm{M}}$ contribution is then retained.

The full procedure for induction quantification from magnetic phase images is detailed in Supplementary Note 5.

Micromagnetic simulations. Micromagnetic simulations were performed using the OOMMF 3D package (http://math.nist.gov/oommf). The universe used for calculation has dimensions of $660 \mathrm{~nm}$ along the $x$ direction, $330 \mathrm{~nm}$ along the $y$ direction and $330 \mathrm{~nm}$ in the $z$ direction (direction parallel to the electron beam) with a size cell of $2 \times 2 \times 2 \mathrm{~nm}$. The simulated FeRh layer has a thickness of $50 \mathrm{~nm}$ along the growth direction ( $y$ direction) and a width (thickness crossed by the electron beam) of $90 \mathrm{~nm}$ from estimations during the FIB process. This width is divided into three layers: a layer of $80 \mathrm{~nm}$ thick composed of ordered FeRh presenting the AFM/FM transition separates two others layers of $5 \mathrm{~nm}$ thick perpendicular to the electron beam corresponding to the amorphized surface of the lamella by the FIB preparation. Three temperatures $\left(25,50\right.$ and $\left.60^{\circ} \mathrm{C}\right)$ have been investigated. The damaged layers were kept in a ferromagnetic state for all applied temperature with a magnetization equal to $1.19 \mathrm{~T}$. This magnetization is oriented along the $x$ direction corresponding to the direction of initial saturation. The volume of ordered FeRh where the magnetization evolves with the temperature is in the form of a rectangular wire with a $50 \times 80 \mathrm{~nm}^{2}$ section and a core-shell structure. The shell takes into account the fact that the magnetic transition at surfaces/interfaces is different from that in the core in which the FM and AFM areas nucleate and coalesce. The shell thickness was taken as the average of the measured widths as a function of temperature (Fig. 4a), and considered to be uniform over the entire section of the layer in the simulations for simplicity. The thickness of the shell and its magnetization parallel to the $x$ direction increase gradually with temperature $(6 \mathrm{~nm} / 0.8 \mathrm{~T}, 9 \mathrm{~nm} / 1 \mathrm{~T}$ and $12 \mathrm{~nm} / 1.19 \mathrm{~T}$ for 25,50 and $60^{\circ} \mathrm{C}$ respectively). The mean magnetization of the FM domains $\left(0.94 \mathrm{~T}\right.$ at $50^{\circ} \mathrm{C}$ and $1.07 \mathrm{~T}$ at $60^{\circ} \mathrm{C}$ ) was extracted from the central portion of Fig. $4 \mathrm{a}$ ) and set parallel to the $x$ direction, while the magnetization of AFM areas is considered equal to 0 . The period of the FM domains is $100 \mathrm{~nm}$ and their lateral size was adjusted accordingly to the experimental phase images $\left(20 \mathrm{~nm}\right.$ to $50^{\circ} \mathrm{C}, 40 \mathrm{~nm}$ to $60^{\circ} \mathrm{C}$ ). From the micromagnetic simulations of this system, the components of the magnetization and the dipolar field have been extracted to calculate the total magnetic induction. After integrating the components perpendicular to the electron beam along the electron path, the corresponding simulated phase image was calculated using Aharonov-Bohm equations and compared directly to experimental magnetic phase images.

Data availability. All relevant data are available from the authors.

\section{References}

1. Fallot, M. Les Alliages du fer avec les métaux de la famille du platine. Ann Phys. (Paris) 10, 291-332 (1938).

2. Fallot, M. \& Hocart, R. Sur l'apparition du ferromagnétisme par élévation de température dans des alliages de fer et de rhodium. Rev. Sci. 77, 498-501 (1939).

3. de Bergevin, F. \& Muldawer, L. Crystallographic study of an iron-rhodium alloy. C. R. Acad. Sci. 252, 1347-1349 (1961).

4. Muldawer, L. \& de Bergevin, F. Antiferromagnetic-ferromagnetic transformation in FeRh. J. Chem. Phys. 35, 1904-1905 (1961).

5. Ibarra, M. R. \& Algarabel, P. A. Giant volume magnetostriction in the FeRh alloy. Phys. Rev. B 50, 4196-4199 (1994).

6. Moruzzi, V. L. \& Marcus, P. M. Antiferromagnetic-ferromagnetic transition in FeRh. Phys. Rev. B 46, 2864-2873 (1992).

7. Gruner, M. E., Hoffmann, E. \& Entel, P. Instability of the rhodium magnetic moment as the origin of the metamagnetic phase transition in $\alpha$-FeRh. Phys. Rev. B 67, 064415 (2003).

8. Gu, R. Y. \& Antropov, V. P. Dominance of the spin-wave contribution to the magnetic phase transition in FeRh. Phys. Rev. B 72, 012403 (2005).

9. Shirane, G., Chen, C. W., Flinn, P. A. \& Nathans, R. Mössbauer study of hyperfine fields and isomer shifts in the Fe-Rh alloys. Phys. Rev. 131, 183-190 (1963).

10. Bordel, C. et al. Fe spin reorientation across the metamagnetic transition in strained FeRh thin films. Phys. Rev. Lett. 109, 117201 (2012).

11. Shirane, G., Nathans, R. \& Chen, C. W. Magnetic moments and unpaired spin densities in the Fe-Rh alloys. Phys. Rev. 134, A1547-A1553 (1964).

12. Fan, R. et al. Ferromagnetism at the interfaces of antiferromagnetic FeRh epilayers. Phys. Rev. B 82, 184418 (2010).

13. Harp, G. R., Parkin, S. S. P., O’Brien, W. L. \& Tonner, B. P. Induced Rh magnetic moments in $\mathrm{Fe}-\mathrm{Rh}$ and $\mathrm{Co}-\mathrm{Rh}$ alloys using $\mathrm{x}$-ray magnetic circular dichroism. Phys. Rev. B 51, 12037-12040 (1995).

14. Ding, Y. et al. Bulk and near-surface magnetic properties of FeRh thin films. J. Appl. Phys. 103, $07 \mathrm{~B} 515$ (2008).

15. Stamm, C. et al. Antiferromagnetic-ferromagnetic phase transition in FeRh probed by x-ray magnetic circular dichroism. Phys. Rev. B 77, 184401 (2008). 
16. Radu, I. et al. Laser-induced generation and quenching of magnetization on FeRh studied with time-resolved x-ray magnetic circular dichroism. Phys. Rev. B 81, 104415 (2010).

17. Baldasseroni, C. et al. Temperature-driven nucleation of ferromagnetic domains in FeRh thin films. Appl. Phys. Lett. 100, 262401 (2012).

18. Mariager, S. O., Guyader, L. L., Buzzi, M., Ingold, G. \& Quitmann, C. Imaging the antiferromagnetic to ferromagnetic first order phase transition of FeRh. Preprint at https://arxiv.org/abs/1301.4164 (2013).

19. Ju, G. et al. Ultrafast generation of ferromagnetic order via a laser-induced phase transformation in FeRh thin films. Phys. Rev. Lett. 93, 197403 (2004).

20. Thiele, J.-U., Maat, S. \& Fullerton, E. E. FeRh/FePt exchange spring films for thermally assisted magnetic recording media. Appl. Phys. Lett. 82, 2859-2861 (2003).

21. Thiele, J. U., Maat, S., Robertson, J. L. \& Fullerton, E. E. Magnetic and structural properties of FePt-FeRh exchange spring films for thermally assisted magnetic recording media. IEEE Trans. Magn. 40, 2537-2542 (2004).

22. Cherifi, R. O. et al. Electric-field control of magnetic order above room temperature. Nat. Mater. 13, 345-351 (2014).

23. Marti, X. et al. Room-temperature antiferromagnetic memory resistor. Nat Mater. 13, 367-374 (2014).

24. Liu, Y. et al. Large reversible caloric effect in FeRh thin films via a dual-stimulus multicaloric cycle. Nat. Commun. 7, 11614 (2016).

25. Kaganer, V. M. et al. Strain-mediated phase coexistence in heteroepitaxial films. Phys. Rev. Lett. 85, 341-344 (2000).

26. Das, A. K. et al. Ferromagnetism of MnAs studied by heteroepitaxial films on GaAs(001). Phys. Rev. Lett. 91, 087203 (2003).

27. Suzuki, I., Koike, T., Itoh, M., Taniyama, T. \& Sato, T. Stability of ferromagnetic state of epitaxially grown ordered FeRh thin films. J. Appl. Phys. 105, 07 E501 (2009).

28. Han, G. C. et al. Magnetic stability of ultrathin FeRh films. J. Appl. Phys. 113, 17C107 (2013).

29. Baldasseroni, C. et al. Effect of capping material on interfacial ferromagnetism in FeRh thin films. J. Appl. Phys. 115, 043919 (2014).

30. Zhou, X., Matthes, F., Bürgler, D. E. \& Schneider, C. M. Magnetic surface domain imaging of uncapped epitaxial FeRh(001) thin films across the temperature-induced metamagnetic transition. AIP Adv. 6, 015211 (2016).

31. Kim, J. W. et al. Surface influenced magnetostructural transition in FeRh films. Appl. Phys. Lett. 95, 222515 (2009).

32. Bennett, S. P. et al. Direct evidence of anomalous interfacial magnetization in metamagnetic Pd doped FeRh thin films. Sci. Rep. 5, 9142 (2015).

33. Bennett, S. P. et al. Giant controllable magnetization changes induced by structural phase transitions in a metamagnetic artificial multiferroic. Sci. Rep. 6, 22708 (2016).

34. Midgley, P. A. \& Dunin-Borkowski, R. E. Electron tomography and holography in materials science. Nat. Mater. 8, 271-280 (2009).

35. Tonomura, A. Applications of electron holography. Rev. Mod. Phys. 59, 639-669 (1987).

36. Gatel, C. et al. Size-specific spin configurations in single iron nanomagnet: from flower to exotic vortices. Nano Lett. 15, 6952-6957 (2015).

37. Reyes, D., Biziere, N., Warot-Fonrose, B., Wade, T. \& Gatel, C. Magnetic configurations in $\mathrm{Co} / \mathrm{Cu}$ multilayered nanowires: evidence of structural and magnetic interplay. Nano Lett. 16, 1230-1236 (2016).

38. Aharonov, Y. \& Bohm, D. Significance of electromagnetic potentials in the quantum theory. Phys. Rev. 115, 485-491 (1959).

39. Aharonov, Y. \& Bohm, D. Further considerations on electromagnetic potentials in the quantum theory. Phys. Rev. 123, 1511-1524 (1961).

40. Yap, Q. J. et al. Phase ordering and its effect on magnetic and structural properties of FeRh ultrathin films. J. Appl. Phys. 116, 043902 (2014).
41. Lounis, S., Benakki, M. \& Demangeat, C. Ferromagnetic stabilization of ordered B2 FeRh thin films. Phys. Rev. B 67, 094432 (2003).

42. Gatel, C. et al. In depth spatially inhomogeneous phase transition in epitaxial MnAs film on GaAs(001). Nano Lett. 17, 2460-2466 (2017).

43. Harada, K., Tonomura, A., Togawa, Y., Akashi, T. \& Matsuda, T. Double-biprism electron interferometry. Appl. Phys. Lett. 84, 3229-3231 (2004).

\section{Acknowledgements}

The authors acknowledge the French National Research Agency under the 'Investissement d'Avenir' programme reference No. ANR-10-EQPX-38-01, the 'Conseil Regional Midi-Pyrénées', the European FEDER for financial support within the CPER programme, and the European Union under the Seventh Framework Program under a contract for an Integrated Infrastructure Initiative Reference 312483-ESTEEM2. This work was supported by the French national project EMMA (ANR12 BS10 013 01) and the French microscopy network METSA.

\section{Author contributions}

C.G. and M.J.C. conceived the research, M.C. deposited epitaxial FeRh thin films and performed VSM measurement, C.G., L.A.R. and D.R. performed the electron holography experiments, C.G. performed the data treatment and analysis, C.G. and N.B. performed micromagnetic simulations, C.G., M.J.C. and B.W.-F. discussed and wrote the manuscript.

\section{Additional information}

Supplementary Information accompanies this paper at http://www.nature.com/ naturecommunications

Competing interests: The authors declare no competing financial interests.

Reprints and permission information is available online at http://npg.nature.com/ reprintsandpermissions/

How to cite this article: Gatel, C. et al. Inhomogeneous spatial distribution of the magnetic transition in an iron-rhodium thin film. Nat. Commun. 8, 15703 doi: 10.1038/ncomms15703 (2017).

Publisher's note: Springer Nature remains neutral with regard to jurisdictional claims in published maps and institutional affiliations.

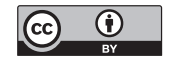

Open Access This article is licensed under a Creative Commons Attribution 4.0 International License, which permits use, sharing, adaptation, distribution and reproduction in any medium or format, as long as you give appropriate credit to the original author(s) and the source, provide a link to the Creative Commons license, and indicate if changes were made. The images or other third party material in this article are included in the article's Creative Commons license, unless indicated otherwise in a credit line to the material. If material is not included in the article's Creative Commons license and your intended use is not permitted by statutory regulation or exceeds the permitted use, you will need to obtain permission directly from the copyright holder. To view a copy of this license, visit http://creativecommons.org/ licenses/by/4.0/

(C) The Author(s) 2017 